\title{
Study of finite amplitude capillary waves stability
}

\author{
Alexander Petrov ${ }^{1,2, *}$ and Mariana Lopushanski ${ }^{2, * *}$ \\ ${ }^{1}$ Institute for Problems in Mechanics RAS, Pr. Vernadskogo 101-1, 119526 Moscow, Russia \\ ${ }^{2}$ Moscow Institute of Physics and Technology, Institutskiy per. 9, 141700 Dolgoprudny, Russia
}

\begin{abstract}
The direct Lyapunov method is used to study capillary waves. The dynamic equations of the capillary wave are presented in the form of an infinite Euler-Lagrange chain of equations for the Stokes coefficients. The stationary solution found for these equations is the Crapper solution for capillary waves. With the help of energy and momentum conservation laws the Lyapunov function is constructed. It is shown that the Lyapunov function is positive definite with respect to any perturbations of waves surfaces with the period that is a multiple of the wave period.
\end{abstract}

\section{Introduction}

In [1] the exact solution of the problem of the potential plane-parallel flow of an ideal fluid in the domain $-\infty<x<\infty,-\infty<y<\eta(k x)$ was built, where the function $\eta(k x)$ is periodic $\eta(k x)=\eta(k x+2 \pi)$, the wave number $k$ is related to the wave length as follows $\lambda=2 \pi / k$. The Laplace condition $p-p_{0}+\sigma / r=0$ is satisfied on the wave surface $\eta(k x)$, where $r$ is the curvature radius of the cylinder, $p$ and $p_{0}$ are the fluid pressure inside and outside the cylinder, $\sigma$ is the surface tension coefficient. Crapper found the exact solution, but used numerical methods to prove it. The solution was expressed through elliptical functions.

The Crapper wave stability was studied in [2]. The infinite chain of linear differential equations for perturbations was obtained and the eigenvalues of these equations were studied. Such a solution requires an excessively large number of computations. The Lagrange method of generalized coordinates may be proposed as an alternative. An analogic method was used in [3] to study the stability of the McLeod plane-parallel flow [4], which models the motion of a drop with surface tension.

The direct Lyapunov method reduces the stability problem to the efficient potential energy minimum condition. The potential energy minimum condition is used to prove the stability of the stationary motion of capillary waves in the frames of the weakened Lyapunov stability definition [5].

In this work the analytic solution for the capillary waves stability problem is presented (earlier numerical methods were used). The second variation of the Lyapunov function to prove the stability of capillary waves with respect to symmetric and non-symmetric disturbances is used.

\footnotetext{
*e-mail: petrovipmech@gmail.com

**e-mail: masha.alexandra@gmail.com
} 


\section{The Lyapunov function}

The dynamics of capillary waves is described, using the wave parametrization introduced by Stokes $[6,7]$. The conformal mapping of the disc $|\zeta|<1$ of the complex plane $\zeta$ with a cut on the positive part of abscissa on the domain of one wave period on the complex plane $z=x+i y$ is considered as follows

$$
z(\zeta)=\frac{\lambda}{2 \pi}\left[i \ln \zeta+\sum_{n=1}^{\infty} z_{n} \zeta^{n}\right]
$$

The circle $\zeta=e^{i \gamma}$ corresponds to the surface of the wave $z=x_{s}+i \eta$. The real and imaginary parts of the Laurent series coefficients $z_{n}=x_{n}+i y_{n}, n=1,2, \ldots$ are considered to be the generalized coordinates of the wave $q_{i}, i=1,2, \ldots$. The motion equations will be the Lagrange equations with the Lagrange function $L$, which equals the difference between the kinetic and the potential wave energy $L=E_{\mathrm{kin}}-E_{\mathrm{pot}}$.

The kinetic energy of the wave is the quadratic function of generalized velocities $\dot{x}_{0}, \dot{q}_{i}, i=1,2, \ldots$, where $x_{0}$ is the cyclic coordinate that determines the horizontal movement of the wave, and $\dot{x}_{0}$ is the wave propagation velocity.

Kinetic energy may be presented as the sum of three types of summands: quadratic in $\dot{x}_{0}$, linear in $x_{0}$ and independent of $\dot{x}_{0}$

$$
E_{k i n}=\frac{1}{2} M \dot{x}_{0}^{2}+M_{1} \dot{x}_{0}+M_{2}=\frac{\left(M \dot{x}_{0}+M_{1}\right)^{2}}{2 M}+M_{*}, \quad M_{*}=M_{2}-\frac{M_{1}^{2}}{2 M} .
$$

Here $M$ is independent of velocities, $M_{1}$ and $M_{2}$ are the linear and quadratic function of velocities $\dot{q}_{i}$. As $E_{k i n}$ is positively definite, then $M_{*}$ is also a positively definite quadratic form of $\dot{q}_{i}$.

Suppose that the system of Lagrange equations has a stationary solution, for which

$$
\dot{x}_{0}=u, \quad \dot{q}_{i}=0, \quad i=1,2, \ldots .
$$

In the solution presented, the surface of the wave moves with velocity $u$, without changing its form. If one considers now the disturbed motion of the wave, the momentum conservation law holds

$$
\frac{\partial E_{\mathrm{kin}}}{\partial \dot{x}_{0}}=M \dot{x}_{0}+M_{1}=M_{0} u
$$

where $M$ is the function of generalized coordinates $q_{i}, M_{0}$ is the value of function $M$ at the stationary point $q_{i}=q_{i}^{0}, i=1,2, \ldots$ Thus, using 2 , the energy conservation law can be written as

$$
\frac{\left(M_{0} u\right)^{2}}{2 M}+E_{\mathrm{pot}}+M_{*}=E .
$$

For stationary motion $M_{*}=0$ and, thus, the energy value is $\frac{\left(M_{0} u\right)}{2}+E_{p o t}^{0}=E_{0}$, where $E_{p o t}^{0}$ is the value of potential energy at a stationary point. Function $E$ is a Lyapunov function if it is positively definite. As $M_{*}$ is positively definite, we consider only the functional $U=$ $\frac{\left(M_{0} u\right)^{2}}{2 M}+E_{p o t}$. If the stationary point is the minimum of $U$, the Lyapunov Theorem implies that the stationary motion is stable.

Consider the system of coordinates in which the fluid is at rest at infinity. The kinetic energy of one period of the stationary wave in this system is expressed through the Stokes coefficients $y_{n}$ as follows [8]

$$
E_{k i n}=\frac{1}{2} M \dot{x}_{0}^{2}, \quad M=\frac{\rho \lambda^{2}}{2 \pi} \frac{S}{2}, \quad S=\sum_{n=1}^{\infty} n\left(x_{n}^{2}+y_{n}^{2}\right) .
$$


The capillary potential energy is proportional to the arc length $l$ of one wave period $E_{\text {pot }}=$ $\sigma l$, where $\sigma$ is the surface tension coefficient. On the complex plane $\zeta$ the arc length is calculated as follows

$$
l=\oint d s=\oint\left|\frac{d z}{d \zeta}\right| \frac{d \zeta}{i \zeta},
$$

where the integral of the differential of the arc length $d s$ is taken along the circle $|\zeta|=1$.

The Stokes coefficients are not very suitable for arc length calculation $l$. So, an analytical function, expressed through parameters $q_{i}$, is introduced

$$
Q(\zeta)=1+\sum_{i=1}^{\infty} q_{i} \zeta^{i}
$$

This function is found using the equality

$$
\begin{gathered}
\frac{d z}{d \zeta}=\frac{\lambda}{2 \pi} \frac{i}{\zeta} Q^{2}(\zeta), \\
\left|\frac{d z}{d \zeta}\right|=\frac{\lambda}{2 \pi} Q(\zeta) \bar{Q}(1 / \zeta), \quad \zeta=e^{i \gamma} .
\end{gathered}
$$

Substituting (5) in (3), the ark length can be written as an integral of this function using the Residue Theorem

$$
l=\frac{\lambda}{2 \pi} \oint Q(\zeta) Q(1 / \zeta) \frac{d \zeta}{i \zeta}=\lambda\left(1+\sum_{n=1}^{\infty}\left|q_{n}\right|^{2}\right)
$$

From (1) and (5) we deduce that

$$
1+\sum_{k=1}^{\infty} k \frac{z_{k}}{i} \zeta^{k}=Q^{2}(\zeta)=1+2 \sum_{k=1}^{\infty} q_{k} \zeta^{k}+\sum_{k=2}^{\infty} \zeta^{k} \sum_{n=1}^{k-1} q_{n} q_{k-n}
$$

and, therefore

$$
z_{1}=2 i q_{1}, \quad k z_{k}=2 i q_{k}+i \sum_{n=1}^{k-1} q_{n} q_{k-n}, \quad k=2,3, \ldots
$$

Using substitution

$$
M=\rho \frac{\lambda^{2}}{2 \pi} \frac{S}{2}, \quad u^{2}=2 \pi \frac{\sigma}{\rho \lambda} \frac{c^{2}}{2 \pi}, \quad l=\frac{\lambda}{2 \pi} \bar{l},
$$

the Lyapunov function can be expressed in the dimensionless form as follows

$$
U=\sigma \frac{\lambda}{2 \pi} \bar{U}, \quad \bar{U}=\frac{S_{0}^{2}}{4 S} c^{2}+\bar{l}, \quad \bar{l}=\frac{l}{\lambda}=1+\sum_{k=1}^{\infty}\left|q_{n}\right|^{2},
$$

where $\bar{U}$ and $\bar{l}$ is a dimensionless Lyapunov function and the arc length of one wave period and $c$ is the dimensionless wave velocity. The assertion that the first variation of $\bar{U}$ equals zero allows finding the parameters $q_{n}$ of the wave and its propagation velocity $c$.

\section{Stationary capillary waves}

It will be shown that the solution of the variational equation $\delta \bar{U}=0$ may be expressed as follows

$$
q_{i}=2 b^{i}
$$

where $b$ is a parameter the solutions family. To prove this small disturbances of coordinates with respect to stationary values are considered

$$
q_{n}=2 b^{n}+\varepsilon\left(\xi_{n}+i \eta_{n}\right), \quad n=1,2, \ldots .
$$


They are substituted into function $\bar{l}(7)$ and expanded by parameter $\varepsilon$

$$
\bar{l}=1+\sum_{k=1}^{\infty}\left(\left(2 b^{n}+\varepsilon \xi_{n}\right)^{2}+\varepsilon^{2} \eta_{n}^{2}\right)=1+\varepsilon \delta \bar{l}+\varepsilon^{2} \delta^{2} \bar{l},
$$

where $\delta \bar{l}$ and $\delta^{2} \bar{l}$ are the first and second variation of $\bar{l}$.

Substituting (9) in (10) and separating the real and imaginary parts $z_{n}=x_{n}+i y_{n}$, the expansion for the Stokes coefficients is found

$$
\begin{gathered}
x_{k}=-\frac{2 \varepsilon}{k}\left(\eta_{k}+2 \sum_{n=1}^{k-1} b^{n} \eta_{k-n}\right), \quad k=1,2, \ldots \\
y_{k}=4 b^{k}+\frac{2 \varepsilon}{k}\left(\xi_{k}+2 \sum_{n=1}^{k-1} b^{n} \xi_{k-n}\right)+\frac{\varepsilon^{2}}{k} \sum_{n=1}^{k-1}\left(\xi_{k-n} \xi_{n}-\eta_{k-n} \eta_{n}\right) .
\end{gathered}
$$

Substituting (refxkyk) in (2), we obtain the expansion by parameter $\varepsilon$ for the functional $S$

$$
S=S_{0}+\varepsilon \delta S+\varepsilon^{2} \delta^{2} S
$$

where $\delta S$ and $\delta^{2} S$ are the first and second variations of $S$.

Substituting expansions (10) and (12) in functional (7) the following expansion $\bar{U}=$ $U_{0}+\varepsilon \delta U+\varepsilon^{2} \delta^{2} U$ is obtained.

The stationary solution is found using the fact that the first variation equals zero

$$
\begin{aligned}
& \delta \bar{U}=-\frac{1}{4} c^{2} \delta S+\delta \bar{l}=0, \quad \delta \bar{l}=\left.\frac{d \bar{l}}{d \varepsilon}\right|_{\varepsilon=0}=4 \Sigma_{1}, \quad \sum_{1}=\sum_{k=1}^{\infty} b^{k} \xi_{k}, \\
& \delta S=\sum_{k=1}^{\infty} 8 b^{k} \delta\left(k y_{k}\right)=16\left(\Sigma_{1}+2 \Sigma_{2}\right), \quad \Sigma_{2}=\sum_{k=1}^{\infty} b^{k} \sum_{n=1}^{k-1} b^{n} \delta q_{k-n} .
\end{aligned}
$$

The double sum $\Sigma_{2}$ may be modified by changing the summation order

$$
\Sigma_{2}=\sum_{n=1}^{\infty} b^{n} \sum_{k=n+1}^{\infty} b^{k} \delta q_{k-n}=\sum_{n=1}^{\infty} b^{2 n} \sum_{k_{1}=1}^{\infty} b^{k_{1}} \delta q_{k_{1}}=\frac{b^{2}}{1-b^{2}} \Sigma_{1} .
$$

This implies that $\delta S=16 \frac{1+b^{2}}{1-b^{2}} \Sigma_{1}$. Substituting $\delta \bar{l}$ and $\delta S$ in equation (13), the equality

$$
-c^{2} 4\left(\frac{1+b^{2}}{1-b^{2}}\right) \Sigma_{1}+4 \Sigma_{1}=0
$$

is obtained, from which wave velocity

$$
c^{2}=\frac{1-b^{2}}{1+b^{2}}
$$

is found. It will be shown that formulas (8) and (14) determine the wave found in [1]. Equality (8) is substituted in (4) and it is obtained that

$$
Q(\zeta)=1+2 \frac{b \zeta}{1-b \zeta}, \quad Q^{2}(\zeta)=\left(\frac{1+b \zeta}{1-b \zeta}\right)^{2}
$$

Thus, by integrating equation (5), it is found that

$$
z(\zeta)=\frac{\lambda}{2 \pi}\left(i \ln \zeta-\frac{4}{1-b \zeta}-4 i\right)
$$


The parameter $b$ may be expressed through the dimensionless amplitude $a$, which is defined as

$$
\frac{\lambda}{2 \pi} a=\frac{1}{2}(z(-i)-z(i))=\frac{\lambda}{2 \pi} \frac{1}{2}\left(\frac{4}{1-b i}-\frac{4}{1+b i}\right) .
$$

Thus the connection between the amplitude and parameter $b$ is found: $a=\frac{4 b}{1-b^{2}}, \quad b=$ $\frac{\sqrt{4+a^{2}}-2}{a}$.

The parametric equation of the wave surface are obtained from (15) for $z=x+i y, \zeta=e^{i \alpha}$

$$
x=-\frac{\lambda}{2 \pi}\left(\alpha+\frac{4 b \sin \alpha}{1-2 b \cos \alpha+b^{2}}\right), \quad y=\frac{\lambda}{2 \pi}\left(\frac{4(1-b \cos \alpha)}{1-2 b \cos \alpha+b^{2}}-4\right) .
$$

These are the same expressions for $x$ and $y$ that were obtained by Crapper. Therefore, a new deduction method for the known exact solution for the capillary wave [1] is presented. The values $b=b_{0}=0.454, a=2.280$ corresponds to the maximum wave development. In Fig. 1 the graphs of waves with values $b:=0.1 ; 0.3$ and maximum wave development $b=0.454$ are presented.

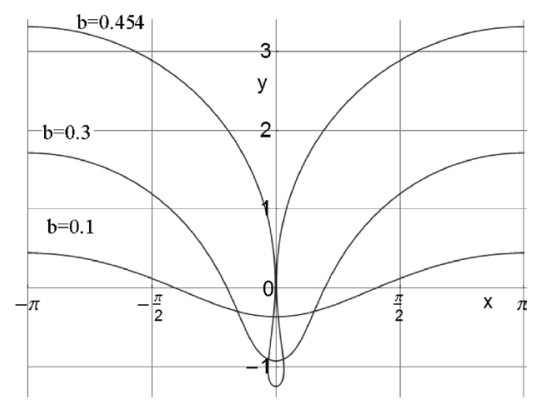

Figure 1. Capillary waves surface at different values of parameter $b$.

\section{Second variation}

The second variation $\delta^{2} \bar{U}=\left.\frac{1}{2} \frac{d^{2} \bar{U}}{d \varepsilon^{2}}\right|_{\varepsilon=0}$ is the quadratic form of variations $\xi_{i}, \eta_{i}, i=1,2, \ldots$ It is expressed through the first and second variations of functionals $S$ and $\bar{l}$. The variables $\xi_{n}$ and $\eta_{n}$ of the second variation $\delta^{2} \bar{U}$ may be separated and the second variation $\delta^{2} \bar{U}$ may be presented as the sum of two quadratic forms $\delta^{2} \bar{U}=\delta^{2} \bar{U}_{1}(\xi)+\delta^{2} \bar{U}_{2}(\eta)$. The first $\delta^{2} \bar{U}_{1}(\xi)$ depends only on $\xi$ and is expressed through $\delta^{2} S_{1}$ and $\delta^{2} \bar{l}_{1}$, which depend only on $\xi$. The second is expressed through $\delta^{2} S_{2}$ and $\delta^{2} \bar{l}_{2}$, which depend only on $\eta$.

The first quadratic form defines the stability of the wave with respect to the symmetric disturbances $\xi_{n}$, the second one defines the stability with respect to the non-symmetric disturbances $\eta_{n}$.

Consider first the quadratic forms of second variations for symmetric disturbances

$$
\delta^{2} \bar{U}_{1}=\frac{c^{2}}{4}\left(\frac{(\delta S)^{2}}{S_{0}}-\delta^{2} S_{1}\right)+\delta^{2} \bar{l}_{1}
$$

For $\delta^{2} U_{1}(\xi)$ the following inequality holds

$$
\delta^{2} \bar{U}_{1}>\lambda_{\min } \sum_{n=1}^{\infty}\left(\xi_{n}\right)^{2}
$$




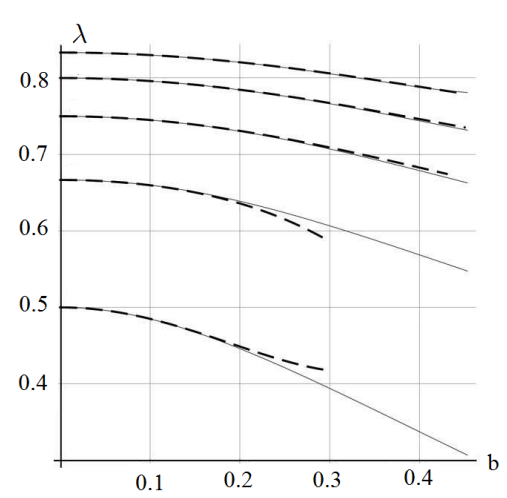

Figure 2. Eigenvalues (symmetric disturbances).

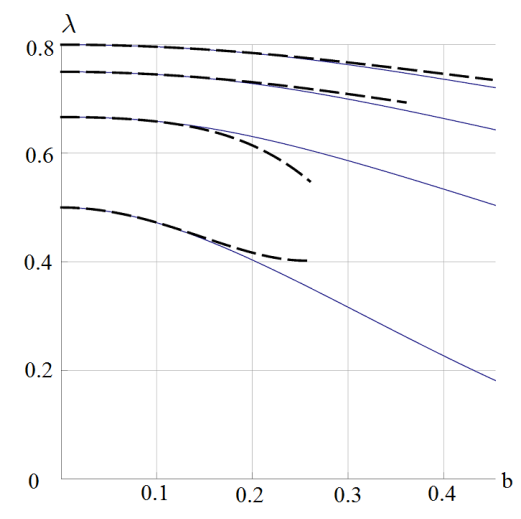

Figure 3. Eigenvalues (non-symmetric disturbances).

where $\lambda_{\min }$ is the smallest eigenvalue of the quadratic form.

The matrix $a_{m n}$ of the quadratic form $\delta^{2} \bar{U}_{1}$ as $b=0$ is diagonal and its diagonal elements are $a_{11}=4, a_{n n}=(n-1) / n, n=2,3, \ldots$. The eigenvalues that correspond to the adjoint linear operator are $\lambda_{n}=a_{n n}$. The smallest eigenvalue is equal to the second diagonal element $\lambda_{\min }=a_{22}=1 / 2$. For eigenvalues the expansion in powers of $b$ may be obtained. For the first five eigenvalues the expansions are

$$
\begin{gathered}
\lambda_{\min }=\lambda_{1}=\frac{1}{2}-\frac{11}{7} b^{2}+\frac{2489}{343} b^{4}, \quad \lambda_{2}=\frac{2}{3}-\frac{2}{3} b^{2}-\frac{38}{15} b^{4}, \\
\lambda_{3}=\frac{3}{4}-\frac{1}{2} b^{2}+\frac{1}{2} b^{4}, \quad \lambda_{4}=\frac{4}{5}-\frac{2}{5} b^{2}+\frac{2}{5} b^{4}, \quad \lambda_{5}=\frac{5}{6}-\frac{1}{3} b^{2}+\frac{1}{3} b^{4} .
\end{gathered}
$$

In Fig. 2 the dependence of the first five eigenvalues on $b$ is presented. The solid lines stand for the results of numeric calculations, the dashed lines stand for the expansions in powers of $b$. From the graphs it is seen that the greater the index of the eigenvalue is, the better it is approximated by its expansion. In the second variation $N=20$ independent variations $\delta q_{i}, \quad i=1,2, \ldots 20$ are taken into consideration. The smallest eigenvalue $\lambda_{1}(b)$ decreases monotonously until it reaches the value $\lambda\left(b_{0}\right)=0.03069$ and for $N>15$ almost does not depend on $N$.

Thus, inequality (16) implies that the second variation $\delta^{2} U>0$ is strictly positive for all variations $\delta q_{i}$. By the Lyapunov Theorem the stationary motion of capillary waves is stable for all possible amplitude values.

The eigenvalues determine the main oscillation frequencies near stationary motion.

Consider now the quadratic form of the second variation for non-symmetric disturbances

$$
\delta^{2} \bar{U}_{2}=-\frac{c^{2}}{4} \delta^{2} S_{2}+\delta^{2} \bar{l}_{2}
$$

The matrix $b_{m n}=\frac{1}{2} \frac{\partial^{2}\left(\delta^{2} \bar{U}_{2}\right)}{\partial \eta_{m} \partial \eta_{n}}$ is diagonal for $b=0$ and $b_{n n}=(n-1) / n, n=2,3, \ldots$ The eigenvalues are $\lambda_{n}=b_{n n}$.

The matrix $b_{m n}$ is singular, its determinant equals zero. This is due to the linear dependence of the generalized $\eta_{1}, \eta_{2}, \eta_{3}, \ldots$, which is expressed as follows

$$
r=\frac{1}{2} \sum_{k=1}^{\infty} k b^{k} \frac{\partial\left(\delta^{2} U_{2}\right)}{\partial \eta_{k}}=0 .
$$


This may be proved as follows. (18) implies that the series $r$ in powers of $b$ is the difference between the series $r_{1}$ and $r_{2}$

$$
\begin{gathered}
r_{1}=\frac{1}{2} \sum_{k=1}^{\infty} k b^{k} \frac{\partial\left(\delta^{2} l_{2}\right)}{\partial \eta_{k}}=\sum_{k=1}^{\infty} k b^{k} \eta_{k}, \\
r_{2}=\frac{1-b^{2}}{8\left(1+b^{2}\right)} \sum_{k=1}^{\infty} k b^{k} \frac{\partial\left(\delta^{2} S_{2}\right)}{\partial \eta_{k}} .
\end{gathered}
$$

It can be checked that in any finite number of coordinates $\eta_{1}, \ldots, \eta_{n}$ the difference $r_{1}-r_{2}$ is small of power $b^{n+1}$

$$
r_{1}-r_{2}=O\left(b^{n+1}\right)
$$

The series $r_{1}$ converges for admissible values of parameter $0<b<0.454$ and bounded values of $\eta_{k}$. The difference of partial sums of $r_{1}$ and $r_{2}$ tends to zero as $n \rightarrow \infty$. Thus the series $r_{2}$ also converges and the difference $r_{1}-r_{2}$ equals zero. Thus we complete the proof.

Equality (19) is equivalent to the fact that the linear combination of the matrix columns $b_{n}$ satisfies the equality $\sum_{k=1}^{\infty} k b^{k} b_{k m}=0$ and, thus, the matrix $\left(b_{m n}\right)$ is singular.

This can also be explained by the fact that the mapping (1) is multivalued. The mapping $\zeta^{\prime}=e^{i \gamma_{0}}$ maps the circle $|\zeta|=1$ into itself, therefore the form of the wave does not change. Therefore, if the first coordinate $\eta_{1}$ is considered to be zero, then all of the other coordinates $\eta_{n}, n=2,3, \ldots$ are independent.

Such choice of coordinates implies that the matrix $\left(b_{m n}\right)$ as $b=0$ is diagonal $b_{m n}=$ $\frac{(n-1)}{n} \delta_{m n}$, and the smallest eigenvalue equals the second diagonal element $\lambda_{\min }=b_{22}=1 / 2$. For eigenvalues we may obtain expansion in powers of $b$. For the first four eigenvalues, set by the increasing index numbers, the expansions are

$$
\begin{gathered}
\lambda_{\text {min }}=\lambda_{1}=\frac{1}{2}-3 b^{2}+23 b^{4} \quad \lambda_{2}=\frac{2}{3}-\frac{2}{3} b^{2}-16 b^{4}, \\
\lambda_{3}=\frac{3}{4}-\frac{1}{2} b^{2}+\frac{1}{2} b^{4}, \quad \lambda_{4}=\frac{4}{5}-\frac{2}{5} b^{2}+\frac{2}{5} b^{4} .
\end{gathered}
$$

In Fig. 3 the dependences of the first four eigenvalues on parameter $b$ are presented. The solid lines stand for the numeric calculations, the dashed ones for the expansion in powers of $b$. The smallest eigenvalue $\lambda_{1}(b)$ monotonously decreases until $\lambda\left(b_{0}\right)=0.181408$ and for the number of generalized coordinates $N>15$ almost does not depend on $N$.

\section{Conclusion}

The analytic solution for the capillary waves stability problem was found. Using the second variation of the Lyapunov function, the stability of capillary waves with respect to symmetric and non-symmetric disturbances was proved.

The study has been supported by the Russian Science Foundation (project N 14-19-01633) in IPMech RAS.

\section{References}

[1] Crapper, G. D., J. Fluid Mech. 2, 532-540 (1957)

[2] Tiron, R., Choi W., J. Fluid Mech. 696, 402 - 422 (2012)

[3] Petrov, A.G., Journal of Applied Mathematics and Mechanics. 3, 366-374 (2016) [in Russian]

[4] McLeod, E.B., J. Ration. Mech. Anal. 4(4), 557-567 (1955)

[5] Lyapunov, A.M., vol. 3, pp. 5-113. Izd. Akad. Nauk SSSR, Moscow (1959) [in Russian]

[6] Sretenskii, L.N.: Theory of Wave Motion of Fluid. Nauka, Moscow (1977) [in Russian]

[7] Petrov, A.G.: Analytical Hydrodynamics. Fizmatlit, Moscow (2009) [in Russian]

[8] Maklakov D.V., Petrov A.G., Doklady RAN. 463(2), 155-159 (2015) 